\title{
The Lie-Poisson Structure of the Euler Equations of an Ideal Fluid
}

\author{
Sergiy Vasylkevych and Jerrold E. Marsden \\ Communicated by Tudor Ratiu, received August 23, 2005
}

\begin{abstract}
This paper provides a precise sense in which the time $t$ map for the Euler equations of an ideal fluid in a region in $\mathbb{R}^{n}$ (or a smooth compact $n$-manifold with boundary) is a Poisson map relative to the Lie-Poisson bracket associated with the group of volume preserving diffeomorphism group. This is interesting and nontrivial because in Eulerian representation, the time $t$ maps need not be $C^{1}$ from the Sobolev class $H^{s}$ to itself (where $s>(n / 2)+1$ ). The idea of how this difficulty is overcome is to exploit the fact that one does have smoothness in the Lagrangian representation and then carefully perform a Lie-Poisson reduction procedure.
\end{abstract}

\section{Introduction}

Hamiltonian structures play a fundamental role in mathematical physics. It's enough to recall a few examples: classical mechanics, electrodynamics, quantum mechanics, hydrodynamics and general relativity. However, when applying the classical methods and technics of symplectic geometry to PDEs, one faces significant difficulties, both analytical and conceptual.

Part of the problem is that symplectic forms that arise in many applications are weak symplectic forms on infinite dimensional manifolds. More importantly, often integral curves of PDEs are not differentiable in time in the function spaces one would normally use; in the linear case, this corresponds to the fact that the operators involved are unbounded. Stock examples include the Euler and Klein-Gordon equations. When dealing with such systems one has to pay careful attention to domains of definitions as many standard formulas become only formal relationships. Their justification is often cumbersome and requires some ad hoc methods.

The goal of this paper is to contribute to the development of techniques that are useful for the treatment of nonlinear PDEs with non-differentiable (in time) solutions and build a framework that allows a systematic and rigorous study of such systems and is applicable to the broad range of physical phenomena. Previous work in this vein is Chernoff and Marsden [1974].

Specifically, this article is devoted to the study of the Euler equations for an ideal fluid on the compact manifold, the example that provides the main inspiration and motivation. The goal is to understand in what exact sense (if any) the flow generated by Euler's equation

1991 Mathematics Subject Classification. Primary: 35; Secondary: 76.

Key words and phrases. Euler equations, Poisson map, Lie-Poisson bracket, Lagrangian representation, Lie-Poisson reduction procedure. 
consists of Poisson maps. Since the classic work of Arnold Arnold [1966], it has been known that formally the Euler equation could be viewed as a Hamiltonian system. (Expositions of this may be found in Arnold and Khesin [1998] and Marsden and Ratiu [1999]).

The work of Ebin and Marsden [1970] showed the remarkable fact that in appropriate function spaces, the flow of the Euler equations in Lagrangian representation (in Sobolev function spaces $H^{s}$ for $\left.s>(n / 2)+1\right)$ is given by a smooth vector field and hence all the difficulties are resolved in that context. This work also shows that one can perform a reduction (Euler-Poincaré reduction) to Eulerian representation to rigorously derive that the solutions obtained this way satisfy the Euler equations (taking into account one derivative loss due to the reduction procedure).

¿From the work of Ebin and Marsden [1970], the reduced flow of the Euler equations in $H^{s}$ are known to form a continuous flow in $H^{s}$ (both in time and in the initial velocity field), and regarded as maps from $H^{s}$ to $H^{s-1}$, they are $C^{1}$. Another remarkable property of the solutions also follows from this same work - namely that the individual particle trajectories are $C^{\infty}$ in time, a fact not so easy to see directly in Eulerian representation (see Kato [2000]).

While a version of the symplectic nature of the flow of the Euler equations follows directly from the results in Ebin and Marsden [1970] (taking into account the loss of one derivative), it is not so clear that there is a well defined Poisson sense for the results. In fact, the work of Lewis, Marsden, Montgomery, and Ratiu [1986] (and many subsequent papers by other authors) shows that in the Poisson context, this derivative loss is a nontrivial issue in defining a good sense in which one has a Poisson manifold and in which the Euler equations then define a Hamiltonian system in the Poisson sense. The main purposes of this paper is to fill this gap by means of a nonsmooth Lie-Poisson reduction procedure on appropriate classes of functions.

This article has the following structure. In $\S 2$ we give important background information on Euler equation and manifolds of diffeomorphisms. Then, we recall the basic ideas of Poisson reduction in $\S 3$. Our results are presented in next two sections. In $\S 4$ we prove that tangent bundle of a weak Riemannian manifold carries a Poisson structure in an appropriate sense, provided that the manifold possesses a smooth Riemannian connection. The later requirement is fulfilled on the groups of diffeomorphisms according to the work of Ebin and Marsden [1970]. In $\S 5$ we utilize this result to show that the flow of Euler equation is Poisson in an appropriate sense. We conclude with short discussion of presented results in $\S 6$.

\section{Solutions of the Euler Equation}

In this section we present some classical results concerning the Euler equation that motivated our study. The notation and exposition follows Ebin and Marsden [1970].

The Euler equations on compact manifold are traditionally formulated in the following way. Let $M$ be a compact Riemannian $n$-manifold possibly with boundary $\partial M$. Find a time dependent vector field $u$, (which has an associated flow denoted $\eta_{t}$ ) such that

(1) $u_{0}$ is a given initial condition with $\operatorname{div} u_{0}=0$

(2) The Euler equations hold:

$$
\frac{\partial u_{t}}{\partial t}+\nabla_{u_{t}} u_{t}=-\operatorname{grad} p_{t}
$$

for some scalar function $p_{t}: M \rightarrow \mathbb{R}$ (the pressure),

(3) $\operatorname{div} u_{t}=0$, and

(4) $u$ is parallel to $\partial M$.

It is standard that above equation can be formally rewritten as an ODE on the space of divergence free vector fields with a derivative loss. But it was discovered by Ebin and Marsden 
[1970] that this is literally true with no derivative loss in Lagrangian representation. We recall how this proceeds. Let $\mu$ be a volume form on the manifold $M$. Let $H^{s}(M, N)$ denote the space of mappings of Sobolev class $s$ from an $n$-manifold $M$ to a manifold $N$. For $s>n / 2+1$, let

$$
\begin{gathered}
\mathcal{D}^{s}=\left\{\eta \in H^{s}(M, M) \mid \eta \text { is bijective and } \eta^{-1} \in H^{s}(M, M)\right\} \text { and } \\
\mathcal{D}_{\mu}^{s}=\left\{\eta \in \mathcal{D}^{s} \mid \eta^{*} \mu=\mu\right\} .
\end{gathered}
$$

Then both $\mathcal{D}^{s}, \mathcal{D}_{\mu}^{s}$ are smooth infinite dimensional manifolds and topological groups, moreover $\mathcal{D}_{\mu}^{s}$ is a closed submanifold and a subgroup of $\mathcal{D}^{s}$.

Let $\widetilde{\tau}: T \mathcal{D}_{\mu}^{s} \rightarrow \mathcal{D}_{\mu}^{s}$ and $\tau: T M \rightarrow M$ be the canonical projections and let $e: M \rightarrow$ $M, \quad e(m)=m$ be the identity element of the groups $\mathcal{D}_{\mu}^{s}, \mathcal{D}^{s}$. Then

$$
\begin{gathered}
T_{\eta} \mathcal{D}^{s}=\left\{u \in H^{s}(M, T M) \mid \tau \circ u=\eta \quad \text { and } u \| \partial M\right\}, \\
T_{e} \mathcal{D}_{\mu}^{s}=\mathfrak{X}_{\operatorname{div}}^{s}(M)=\left\{u \in H^{s}(M, T M) \mid \tau \circ u=e, \operatorname{div} u=0 \text { and } u \| \partial M\right\},
\end{gathered}
$$

where $\mathfrak{X}_{\text {div }}^{s}(M)$ denotes the space of $H^{s}$ divergence free vector fields on $M$ that are parallel to the boundary.

A given Riemannian metric on $M$ induces a right invariant weak Riemannian metric on $\mathcal{D}_{\mu}^{s}$ given by

$$
\langle X, Y\rangle_{\eta}=\int_{M}\langle X(m), Y(m)\rangle_{\eta(m)} \mu(m)
$$

for $X, Y \in T_{\eta} \mathcal{D}_{\mu}^{s}$ where scalar product under the integral sign is taken in $M$.

As was shown in Ebin and Marsden [1970], $\mathcal{D}_{\mu}^{s}$ possesses a smooth Riemannian connection and, as a consequence, a smooth spray, which we will denote $S$.

Proposition 2.1. (Ebin and Marsden [1970]) For $s>(n / 2)+1$, the weak Riemannian metric (2.2) has a $C^{\infty}$ spray $S: T \mathcal{D}_{\mu}^{s} \rightarrow T T \mathcal{D}_{\mu}^{s}$. Let $F_{t}: T \mathcal{D}_{\mu}^{s} \rightarrow T \mathcal{D}_{\mu}^{s}$ be the (local, $C^{\infty}$ ) flow of $S$. Let $v_{t}=F_{t}\left(u_{0}\right)$ (the material velocity field) and $\eta_{t}=\tilde{\tau}\left(v_{t}\right)$ (the particle position field). Then the solution of the Euler equation with initial condition $u(0)=u_{0}$ is given by

$$
u_{t}=v_{t} \circ \eta_{t}^{-1}
$$

¿From the properties of the diffeomorphism group, one sees that this result shows that the Euler equations (2.1) are well-posed in $H^{s}$ in Eulerian representation.

\section{Motivation: The Poisson Reduction Theorem}

First, recall the following basic and simple result about Poisson reduction (see, for example, Marsden and Ratiu [1999]).

Suppose that $G$ is a Lie group that acts on a Poisson manifold $P$ and that for each $g \in G$ the action map $\Phi_{g}: P \rightarrow P$ is a Poisson map. Suppose that the quotient $P / G$ is a smooth manifold and the projection $\pi: P \rightarrow P / G$ is a submersion. Then, there is a unique Poisson structure $\{\cdot, \cdot\}$ on $P / G$ such that $\pi$ is a Poisson map. It is given by

$$
\{f, k\} \circ \pi=\{f \circ \pi, k \circ \pi\}_{P} \quad \forall k, f \in \mathcal{F}(P / G),
$$

where $\{\cdot, \cdot\}_{P}$ is a Poisson bracket in $P$ and $\mathcal{F}(P / G)$ is a set of smooth functions on $P / G$.

If $\mathbf{X}_{H}$ is a Hamiltonian vector field for a G-invariant Hamiltonian $H \in \mathcal{F}(P)$, then $\pi$ also induces reduction of dynamics. There is a function $h \in \mathcal{F}(P / G)$ such that $H=h \circ \pi$. Since $\pi$ is a Poisson map it transforms $\mathbf{X}_{H}$ on $P$ to $\mathbf{X}_{h}$ on $P / G$, that is, $T \pi \circ \mathbf{X}_{H}=\mathbf{X}_{h} \circ \pi$. 
Denoting the flow of $\mathbf{X}_{H}$ by $F_{t}$ and the flow of $\mathbf{X}_{h}$ by $\tilde{F}_{t}$ we obtain commutative diagram

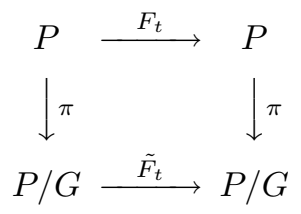

Our strategy is to apply the above procedure to the context of fluids. To do so, define the map $\pi: T \mathcal{D}_{\mu}^{s} \rightarrow \mathfrak{X}_{\text {div }}^{s}$ via

$$
\pi(\eta, v)=v \circ \eta^{-1},
$$

where $\eta \in \mathcal{D}_{\mu}^{s} ;(\eta, v) \in T_{\eta} \mathcal{D}_{\mu}^{s} ; \tau \circ v=\eta$. Let $\tilde{F}_{t}: \mathfrak{X}_{\text {div }}^{s} \rightarrow \mathfrak{X}_{\text {div }}^{s}$ be given by

$$
\tilde{F}_{t}(v)=\pi \circ F_{t}(v)
$$

for $v \in \mathfrak{X}_{\text {div }}^{s}$. By Proposition 2.1, $\tilde{F}_{t}$ is the flow of Euler equation on $\mathfrak{X}_{\text {div }}^{s}$, i.e. $u_{t}=\tilde{F}_{t}\left(u_{0}\right)$ satisfies the Euler equations (2.1).

It is clear from the preceding developments that $F_{t}$ (as a flow of a spray) is a flow of Hamiltonian vector field on $T \mathcal{D}_{\mu}^{s}$. The following commutative diagram

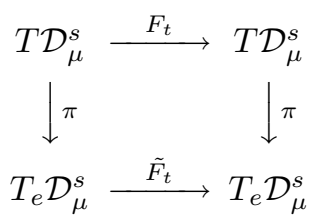

suggests that the flow of Euler equation itself, which is obtained from $F_{t}$ via Poisson reduction, should be a Hamiltonian flow in the sense of Poisson manifolds and this is certainly formally true (see, for instance Lewis, Marsden, Montgomery, and Ratiu [1986] for both the case considered here as well as the case of free boundary problems).

However, as noted in this reference and elsewhere, there are difficulties in finding the right class of functions so that one gets a Poisson structure in a precise sense. To justify the formal insight in precise function spaces, one has to overcome two hurdles.

The first hurdle is that $T \mathcal{D}_{\mu}^{s}$ is only a weak symplectic manifold, and therefore does not necessary carry a Poisson bracket in any obvious way without special ad hoc hypotheses such as "the needed functional derivatives exist" which have long been recognized as awkward at best.

The second hurdle is that $T \mathcal{D}_{\mu}^{s}$ is not a Lie group in the usual sense (left multiplication is not smooth), and $\pi$ is not a smooth map (inversion in $\mathcal{D}_{\mu}^{s}$ is not smooth). Therefore, the well developed theory of Poisson and Lie-Poisson reduction is not directly applicable in this case, even though the loss of derivatives one suffers from these transformations is well understood.

The main point of this paper is to resolve these difficulties in what we believe is a satisfactory way. We do this in the following sections.

\section{Poisson Structures on Weak Riemannian Manifolds}

Let $Q$ be a weak Riemannian manifold modelled on Banach space $\mathbf{E}$ with metric $\langle\cdot, \cdot\rangle$. Then $T Q$ possesses a canonical weak symplectic form that is given in charts by the following standard formula (see, e.g., Marsden and Ratiu [1999]):

$$
\Omega(\eta, e)\left(\left(e_{1}, e_{2}\right),\left(e_{3}, e_{4}\right)\right)=\left\langle e_{1}, e_{4}\right\rangle_{\eta}-\left\langle e_{2}, e_{3}\right\rangle_{\eta}+D_{\eta}\left\langle e, e_{1}\right\rangle_{\eta} \cdot e_{3}-D_{\eta}\left\langle e, e_{3}\right\rangle_{\eta} \cdot e_{1},
$$

where $\eta \in Q, e, e_{1}, e_{2}, e_{3}, e_{4} \in E$. 
For a smooth function $f: M \rightarrow \mathbb{R}$ on a (strong) symplectic manifold $\left(M, \Omega_{1}\right)$, let $\mathbf{X}_{f}$ denote its Hamiltonian vector field. Then

$$
\{f, g\}=\Omega_{1}\left(X_{f}, X_{g}\right)
$$

makes $(M,\{\cdot, \cdot\})$ into a Poisson manifold.

Since $\Omega$ is weak, formula 4.1 does not automatically define Poisson bracket $\{f, g\}$ for arbitrary functions $f, g \in \mathcal{F}(T Q)$ since $\mathbf{X}_{f}, \mathbf{X}_{g}$ may fail to exist and even if they do, one has to make additional hypotheses to obtain the Jacobi identity.

However, under the two additional hypothesis:

(1) $Q$ has smooth Riemannian connection;

(2) The inclusion $T_{\eta} Q \rightarrow T_{\eta}^{*} Q$ (the literal dual space) via

$$
v(u)=\langle v, u\rangle_{\eta} \quad \forall u \in T_{\eta} Q
$$

is dense,

it will be shown that one can define a Poisson bracket on the subalgebra

$$
\mathcal{K}(T Q)=\left\{f \in T Q \mid \frac{\partial f}{\partial \eta}, \frac{\partial f}{\partial v} \in C^{\infty}(T Q, T Q)\right\}
$$

of $\mathcal{F}(T Q)$. Here $\frac{\partial f}{\partial \eta}, \frac{\partial f}{\partial v}$ are covariant partial derivatives on $T Q$, the definition of which will be given below.

This newly defined bracket makes $\mathcal{K}(T Q)$ into a Lie algebra and retains essential dynamical properties of a "true" Poisson bracket, including the Jacobi identity and the fact that flows of Hamiltonian vector fields are Poisson maps and, of course, energy is conserved. Moreover, we will show that the bracket indeed is related to the canonical weak symplectic form in the way that one would expect. In the following we assume that conditions (1) and (2) are satisfied.

Covariant Partial Derivatives. First, we introduce covariant partial derivatives on $T Q$. Let $\tau: T Q \rightarrow Q$ and $\tau_{1}: T T Q \rightarrow T Q$ be natural projections, $\Gamma: Q \supset U \times E \times E \rightarrow E$ be a Christoffel map and $K: T T Q \rightarrow T Q$ be a connector map. In local representation,

$$
K(\eta, v, u, w)=(\eta, w+\Gamma(\eta)(v, u)) .
$$

Define $\Theta: T T Q \rightarrow T Q \oplus T Q \oplus T Q$ by

$$
\Theta=\left(\tau_{1}, T \tau, K\right) .
$$

It is standard that $\Theta$ is a diffeomorphism (see Eliasson [1967]). For $H: T Q \rightarrow \mathbb{R}$ we set

$$
\begin{array}{ll}
\frac{\partial H}{\partial \eta}(V) \cdot W=d H \cdot \Theta^{-1}(V, W, 0) & \forall V, W \in T_{q} Q, \\
\frac{\partial H}{\partial v}(V) \cdot W=d H \cdot \Theta^{-1}(V, 0, W) & \forall V, W \in T_{q} Q .
\end{array}
$$

In local representation, this reads

$$
\begin{aligned}
\frac{\partial H}{\partial \eta}(\eta, v) \cdot(\eta, u) & =d H \cdot \Theta^{-1}((\eta, v),(\eta, u),(\eta, 0)) \\
& =d H \cdot(\eta, v, u,-\Gamma(\eta)(v, u)),
\end{aligned}
$$

and

$$
\begin{aligned}
\frac{\partial H}{\partial v}(\eta, v) \cdot(\eta, w) & =d H \cdot \Theta^{-1}((\eta, v),(\eta, 0),(\eta, w)) \\
& =d H \cdot(\eta, v, 0, w)) .
\end{aligned}
$$


Similarly, for $\phi: T Q \rightarrow T Q_{1}$ we define $\frac{\partial \phi}{\partial \eta}, \frac{\partial \phi}{\partial v}: T Q \rightarrow L\left(T Q, T T Q_{1}\right)$ (here $L\left(T Q, T T Q_{1}\right)$ is the space of linear maps $T Q \rightarrow T T Q_{1}$ ) by

$$
\begin{array}{ll}
\frac{\partial \phi}{\partial \eta}(V) \cdot W=T \phi \cdot \Theta^{-1}(V, W, 0) & \forall V, W \in T_{q} Q, \\
\frac{\partial \phi}{\partial v}(V) \cdot W=T \phi \cdot \Theta^{-1}(V, 0, W) & \forall V, W \in T_{q} Q .
\end{array}
$$

The following Lemmas are readily verified.

Lemma 4.1. Let $X$ be a vector field on $T Q, Y$ be a vector field on $T Q_{1}, \phi: T Q_{1} \rightarrow T Q$. Then

$$
\begin{aligned}
d H \cdot X & =\frac{\partial H}{\partial \eta} \cdot T \tau(X)+\frac{\partial H}{\partial v} \cdot K(X), \\
\frac{\partial(H \circ \phi)}{\partial \eta} \cdot Y & =d H \cdot\left(\frac{\partial \phi}{\partial \eta} \cdot Y\right), \\
\frac{\partial(H \circ \phi)}{\partial v} \cdot Y & =d H \cdot\left(\frac{\partial \phi}{\partial v} \cdot Y\right) .
\end{aligned}
$$

Lemma 4.2. For $H \in C^{1}(T Q, \mathbb{R})$, we have

$$
\begin{aligned}
& \frac{\partial H}{\partial \eta}(\eta, v) \cdot(\eta, u)=\left.\frac{d}{d t}\right|_{t=0} H\left(\eta_{t}, v_{t}\right), \\
& \frac{\partial H}{\partial v}(\eta, v) \cdot(\eta, w)=\left.\frac{d}{d t}\right|_{t=0} H(\eta, v+t w),
\end{aligned}
$$

where $\left(\eta_{t}, v_{t}\right)$ is the parallel translation of $(\eta, v)$ along the curve $\eta_{t}$ with $\eta_{t}^{\prime}(0)=u$.

Let

$$
\mathcal{K}^{k}(T Q)=\left\{f \in C^{k+1}(T Q, \mathbb{R}) \mid \frac{\partial f}{\partial \eta}, \frac{\partial f}{\partial v} \in C^{k}(T Q, T Q)\right\} .
$$

Now we can define the bracket $\{\cdot, \cdot\}$ via

$$
\{f, g\}(\eta, v)=\left\langle\frac{\partial f}{\partial \eta}(\eta, v), \frac{\partial g}{\partial v} v(\eta, v)\right\rangle_{\eta}-\left\langle\frac{\partial f}{\partial v}(\eta, v), \frac{\partial g}{\partial \eta}(\eta, v)\right\rangle_{\eta} .
$$

Preliminaries on the Poisson Structure. The following is the first main result.

TheOREM 4.3. The bracket (4.2) maps $\mathcal{K}^{k} \times \mathcal{K}^{m}$ into $\mathcal{K}^{\min (k, m)-1}$ and also maps $\mathcal{K} \times \mathcal{K}$ into $\mathcal{K}$.

Remark. By definition of the covariant partial derivatives, $\frac{\partial h}{\partial \eta}, \frac{\partial h}{\partial v}: T_{\eta} Q \rightarrow T_{\eta}^{*} Q$ for $h: T Q \rightarrow \mathbb{R}$. The theorem asserts that if $h=\{f, g\}$ then, in fact, $\frac{\partial h}{\partial \eta}(\eta, v), \frac{\partial h}{\partial v}(\eta, v) \in T Q$, i.e. there are $Z(\eta, v), Y(\eta, v) \in T_{\eta} Q$ such that

$$
\frac{\partial h}{\partial \eta}(\eta, v) \cdot X=\langle Z, X\rangle, \quad \frac{\partial h}{\partial v}(\eta, v) \cdot X=\langle Z, X\rangle \quad \forall X \in T_{\eta} Q
$$

and the maps $(\eta, v) \rightarrow Z(\eta, v), Y(\eta, v)$ have appropriate smoothness. 
Proof. Define operator $\frac{\mathrm{D}}{d t}=K \circ \frac{d}{d t}$. This definition extends the usual notion of covariant derivative from vector fields along curves on $Q$ to arbitrary curves on $T Q$. Let $f, g: T Q \rightarrow \mathbb{R}$ and $h=\{f, g\}$. Choosing $\left(\eta_{t}, v_{t}\right)$ as in Lemma 4.2 , we obtain

$$
\begin{aligned}
& \frac{\partial h}{\partial \eta}(\eta, v) \cdot(\eta, u)\left.\frac{d}{d t}\right|_{t=0}\left\langle\frac{\partial f}{\partial \eta}\left(\eta_{t}, v_{t}\right), \frac{\partial g}{\partial v}\left(\eta_{t}, v_{t}\right)\right\rangle_{\eta_{t}}-\left.\frac{d}{d t}\right|_{t=0}\left\langle\frac{\partial f}{\partial v}\left(\eta_{t}, v_{t}\right), \frac{\partial g}{\partial \eta}\left(\eta_{t}, v_{t}\right)\right\rangle_{\eta_{t}} \\
&=\left\langle\left.\frac{\mathbf{D}}{d t}\right|_{t=0} \frac{\partial f}{\partial \eta}\left(\eta_{t}, v_{t}\right), \frac{\partial g}{\partial v}(\eta, v)\right\rangle_{\eta}+\left\langle\frac{\partial f}{\partial \eta}(\eta, v),\left.\frac{\mathbf{D}}{d t}\right|_{t=0} \frac{\partial g}{\partial v}\left(\eta_{t}, v_{t}\right)\right\rangle_{\eta} \\
& \quad-\left\langle\left.\frac{\mathbf{D}}{d t}\right|_{t=0} \frac{\partial f}{\partial v}\left(\eta_{t}, v_{t}\right), \frac{\partial g}{\partial \eta}(\eta, v)\right\rangle_{\eta}-\left\langle\frac{\partial f}{\partial v}(\eta, v),\left.\frac{\mathbf{D}}{d t}\right|_{t=0} \frac{\partial g}{\partial \eta}\left(\eta_{t}, v_{t}\right)\right\rangle_{\eta} \\
&=\left\langle K \frac{\partial}{\partial \eta} \frac{\partial f}{\partial \eta}(\eta, v) \cdot(\eta, u), \frac{\partial g}{\partial v}(\eta, v)\right\rangle_{\eta}+\left\langle K \frac{\partial}{\partial \eta} \frac{\partial g}{\partial v}(\eta, v) \cdot(\eta, u), \frac{\partial f}{\partial \eta}(\eta, v)\right\rangle_{\eta} \\
& \quad-\left\langle K \frac{\partial}{\partial \eta} \frac{\partial g}{\partial \eta}(\eta, v) \cdot(\eta, u), \frac{\partial f}{\partial v}(\eta, v)\right\rangle_{\eta}-\left\langle K \frac{\partial}{\partial \eta} \frac{\partial f}{\partial v}(\eta, v) \cdot(\eta, u), \frac{\partial g}{\partial \eta}(\eta, v)\right\rangle_{\eta} .
\end{aligned}
$$

To proceed further, we need to calculate the quantity

$$
\left\langle K \frac{\partial}{\partial \eta} \frac{\partial f}{\partial \eta}(\eta, v) \cdot(\eta, u),(\eta, w)\right\rangle_{\eta}
$$

where $(\eta, w)$ is an arbitrary element of $T_{\eta} Q$. Let $\left(\eta_{t s}, v_{t s}\right)$ be a parametric surface in $T Q$ with the following properties:

(1) $\left.\frac{d}{d t}\right|_{t=0} \eta_{t 0}=u,\left(\eta_{00}, v_{00}\right)=(\eta, v)$;

(2) $\left(\eta_{t 0}, v_{t 0}\right)$ is a parallel translation of $(\eta, v)$;

(3) $\left(\eta_{t 0}, w_{t}\right)$ is a parallel translation of $\left(\eta_{00}, w_{0}\right)=(\eta, w)$;

(4) $\left.\frac{d}{d s}\right|_{s=0} \eta_{t s}=w_{t}$ for all $s$;

(5) $\left(\eta_{t s}, v_{t s}\right)$ is a parallel translation of $\left(\eta_{t 0}, v_{t 0}\right)$ for all $s$.

Then, keeping in mind Lemmas 4.1, 4.2 and symmetry of Riemannian connection, one checks the following:

$$
\begin{gathered}
\left\langle K \frac{\partial}{\partial \eta} \frac{\partial f}{\partial \eta}(\eta, v) \cdot(\eta, u),(\eta, w)\right\rangle_{\eta}=\left\langle\left.\frac{\mathbf{D}}{d t}\right|_{t=0} \frac{\partial f}{\partial \eta}\left(\eta_{t 0}, v_{t 0}\right),(\eta, w)\right\rangle_{\eta} \\
=\frac{d}{d t}{ }_{t=0}\left\langle\frac{\partial f}{\partial \eta}\left(\eta_{t 0}, v_{t 0}\right),\left(\eta_{t 0}, w_{t}\right)\right\rangle_{\eta_{t}} \\
=\left.\frac{d}{d t}\right|_{t=0} \frac{d}{d s}{ }_{s=0} f\left(\eta_{t s}, v_{t s}\right)=\frac{d}{d s}{ }_{s=0} \frac{d}{d t}{ }_{t=0} f\left(\eta_{t s}, v_{t s}\right)=\left.\frac{d}{d s}{ }_{s=0} d f \cdot \frac{d}{d t}\right|_{t=0}\left(\eta_{t s}, v_{t s}\right) \\
=\left.\frac{d}{d s}\right|_{s=0}\left[\frac{\partial f}{\partial v}\left(\eta_{0 s}, v_{0 s}\right) \cdot K \frac{d}{d t} t=0\right. \\
=\left.\frac{d}{d s}\right|_{s=0}\left[\left\langle\frac{\partial f}{\partial v}\left(\eta_{t s}, v_{t s}\right)+\frac{\partial f}{\partial \eta}\left(\eta_{0 s}, v_{0 s}\right) \cdot T \tau \frac{d}{d t} v_{t=0}\left(\eta_{t s}, v_{t s}\right)\right]\right. \\
\left.\left.\left.=\left\langle\frac{\mathbf{D}}{d t}{ }_{t=0}\left(\eta_{t s}, v_{t s}\right)\right\rangle_{\eta 0 s}+\left\langle\frac{\partial f}{\partial \eta}\left(\eta_{0 s}, v_{0 s}\right), \frac{\mathbf{D}}{d t} \eta_{t=0}\left(\eta_{t 0}, v_{t 0}\right)\right\rangle_{\eta s}\right), \frac{d}{d t}{ }_{t=0} \eta_{t s}\right\rangle_{\eta_{0 s}}\right] \\
+\left\langle\frac{\mathbf{D}}{\partial v}(\eta, v),\left.\frac{\mathbf{D}}{d s}{ }_{s=0} \frac{\mathbf{D}}{d t}\right|_{t=0}\left(\eta_{t s}, v_{t s}\right)\right\rangle_{\eta} \\
\left.\quad\left(\eta_{0 s}, v_{0 s}\right), \frac{d}{d t}{ }_{t=0} \eta_{t 0}\right\rangle_{\eta}+\left\langle\frac{\partial f}{\partial \eta}(\eta, v),\left.\frac{\mathbf{D}}{d s}\right|_{s=0} \frac{d}{d t}{ }_{t=0} \eta_{t s}\right\rangle_{\eta} .
\end{gathered}
$$


Lemma 4.4. (see Do Carmo [1992]). Let $\mathcal{R}$ denote the Ricci curvature tensor. Then

$$
\begin{gathered}
\frac{\mathbf{D}}{d s} \frac{\mathbf{D}}{d t}\left(\eta_{t s}, v_{t s}\right)=\frac{\mathbf{D}}{d t} \frac{\mathbf{D}}{d s}\left(\eta_{t s}, v_{t s}\right)+\mathcal{R}\left(\frac{d}{d t} \eta_{t s}, \frac{d}{d s} \eta_{t s}\right)\left(\eta_{t s}, v_{t s}\right), \\
\frac{\mathbf{D}}{d s} \frac{d}{d t} \eta_{t s}=\frac{\mathbf{D}}{d t} \frac{d}{d s} \eta_{t s} .
\end{gathered}
$$
obtain

By construction of $\left(\eta_{t s}, v_{t s}\right)$, we have $\left.\frac{\mathrm{D}}{d t}\right|_{t=0}\left(\eta_{t 0}, v_{t 0}\right)=0$. Applying lemma 4.4 we

$$
\begin{gathered}
\left.\frac{\mathbf{D}}{d s}{ }_{s=0} \frac{\mathbf{D}}{d t}\right|_{t=0}\left(\eta_{t s}, v_{t s}\right)=\mathcal{R}\left(\left.\frac{d}{d t}\right|_{t=0} \eta_{t 0},\left.\frac{d}{d s}\right|_{s=0} \eta_{0 s}\right)(\eta, v)=\mathcal{R}((\eta, u),(\eta, w))(\eta, v), \\
\left.\frac{\mathbf{D}}{d s}{ }_{s=0} \frac{d}{d t}\right|_{t=0} \eta_{t s}=\frac{\mathbf{D}}{d t}{ }_{t=0}\left(\eta_{t 0}, w_{t}\right)=0 .
\end{gathered}
$$

Thus,

$$
\begin{aligned}
&\langle K\left.\frac{\partial}{\partial \eta} \frac{\partial f}{\partial \eta}(\eta, v) \cdot(\eta, u),(\eta, w)\right\rangle_{\eta} \\
&=0+\left\langle\frac{\partial f}{\partial v}(\eta, v), \mathcal{R}((\eta, u),(\eta, w))(\eta, v)\right\rangle_{\eta} \\
&+\left\langle K \frac{\partial}{\partial \eta} \frac{\partial f}{\partial \eta}(\eta, v) \cdot(\eta, w),(\eta, u)\right\rangle_{\eta}+0 \\
&=\left\langle K \frac{\partial}{\partial \eta} \frac{\partial f}{\partial \eta}(\eta, v) \cdot(\eta, w),(\eta, u)\right\rangle_{\eta}-\left\langle\mathcal{R}\left((\eta, v), \frac{\partial f}{\partial v}(\eta, v)\right)(\eta, w),(\eta, u)\right\rangle_{\eta}
\end{aligned}
$$

by Bianchi's identity. Similar calculations yield

$$
\begin{aligned}
& \left\langle K \frac{\partial}{\partial v} \frac{\partial f}{\partial v}(\eta, v) \cdot(\eta, u),(\eta, w)\right\rangle_{\eta}=\left\langle K \frac{\partial}{\partial v} \frac{\partial f}{\partial v}(\eta, v) \cdot(\eta, w),(\eta, u)\right\rangle_{\eta}, \\
& \left\langle K \frac{\partial}{\partial \eta} \frac{\partial f}{\partial v}(\eta, v) \cdot(\eta, u),(\eta, w)\right\rangle_{\eta}=\left\langle K \frac{\partial}{\partial v} \frac{\partial f}{\partial \eta}(\eta, v) \cdot(\eta, w),(\eta, u)\right\rangle_{\eta} .
\end{aligned}
$$
get

Substituting this into the formulas for $\frac{\partial h}{\partial \eta}$ and using Bianchi's identity once again, we

$$
\begin{aligned}
& \frac{\partial h}{\partial \eta}(\eta, v) \cdot(\eta, u)=\left\langle K \frac{\partial}{\partial \eta} \frac{\partial f}{\partial \eta}(\eta, v) \cdot \frac{\partial g}{\partial v}(\eta, v)+K \frac{\partial}{\partial v} \frac{\partial g}{\partial \eta}(\eta, v) \cdot \frac{\partial f}{\partial \eta}(\eta, v),(\eta, u)\right\rangle_{\eta} \\
& -\left\langle K \frac{\partial}{\partial \eta} \frac{\partial g}{\partial \eta}(\eta, v) \cdot \frac{\partial f}{\partial v}(\eta, v)+K \frac{\partial}{\partial v} \frac{\partial f}{\partial \eta}(\eta, v) \cdot \frac{\partial g}{\partial \eta}(\eta, v),(\eta, u)\right\rangle_{\eta} \\
& \quad+\left\langle\mathcal{R}\left(\frac{\partial f}{\partial v}, \frac{\partial g}{\partial \eta}\right) \cdot(\eta, v),(\eta, u)\right\rangle_{\eta} .
\end{aligned}
$$

Similarly,

$$
\begin{gathered}
\frac{\partial h}{\partial v}(\eta, v) \cdot(\eta, u)=\left\langle K \frac{\partial}{\partial \eta} \frac{\partial f}{\partial v}(\eta, v) \cdot \frac{\partial g}{\partial v}(\eta, v)+K \frac{\partial}{\partial v} \frac{\partial g}{\partial v}(\eta, v) \cdot \frac{\partial f}{\partial \eta}(\eta, v),(\eta, u)\right\rangle_{\eta} \\
-\left\langle K \frac{\partial}{\partial \eta} \frac{\partial g}{\partial v}(\eta, v) \cdot \frac{\partial f}{\partial v}(\eta, v)+K \frac{\partial}{\partial v} \frac{\partial f}{\partial v}(\eta, v) \cdot \frac{\partial g}{\partial \eta}(\eta, v),(\eta, u)\right\rangle_{\eta}
\end{gathered}
$$

As $K$ is smooth, the statement of the theorem follows. 
Hamiltonian Vector Fields. The smoothness structure of Hamiltonian vector fields is given as follows.

Proposition 4.5. The vector field $\mathbf{X}_{H}$ is a $C^{k}$ Hamiltonian vector field (with respect to canonical weak symplectic form) on $T Q$ of class $C^{k}$ if and only if $H \in \mathcal{K}^{k}(T Q)$. Moreover,

$$
\mathbf{X}_{H}(\eta, v)=\left(\eta, v, \frac{\partial H}{\partial v},-\frac{\partial H}{\partial \eta}-\Gamma(\eta)\left(v, \frac{\partial H}{\partial v}\right)\right)
$$

Proof. In local representation, we have

$$
\Omega(\eta, e)\left(\left(e_{1}, e_{2}\right),\left(e_{3}, e_{4}\right)\right)=\left\langle e_{1}, e_{4}\right\rangle_{\eta}-\left\langle e_{2}, e_{3}\right\rangle_{\eta}+\left\langle\Gamma(\eta)\left(e, e_{3}\right), e_{1}\right\rangle_{\eta}-\left\langle\Gamma(\eta)\left(e, e_{1}\right), e_{3}\right\rangle_{\eta} .
$$

Indeed,

$$
D_{\eta}\left\langle e, e_{1}\right\rangle \cdot e_{3}=\left\langle\Gamma(\eta)\left(e_{3}, e_{1}\right), e\right\rangle_{\eta}+\left\langle\Gamma(\eta)\left(e_{3}, e\right), e_{1}\right\rangle_{\eta} .
$$

Substituting this expression into the formula for $\Omega$ and using the symmetry of $\Gamma$ we obtain the desired result.

Let $\mathbf{X}_{H}=\left(\eta, v, e_{1}, e_{2}\right)$ be a Hamiltonian vector field, $Z=(\eta, v, u, w) \in T_{(\eta, v)} T Q$ be arbitrary. Then

$$
\Omega\left(\mathbf{X}_{H}, Z\right)=\left\langle w+\Gamma(\eta)(v, u), e_{1}\right\rangle-\left\langle e_{2}+\Gamma(\eta)\left(v, e_{1}\right), u\right\rangle .
$$

On the other hand, by lemma 4.1

$$
\Omega\left(\mathbf{X}_{H}, Z\right)=d H \cdot Z=\frac{\partial H}{\partial \eta} \cdot T \tau Z+\frac{\partial H}{\partial v} K Z=\frac{\partial H}{\partial \eta} \cdot(\eta, u)+\frac{\partial H}{\partial v} \cdot(\eta, w+\Gamma(u, v)) .
$$

Setting $u=0$ and comparing the above expressions we see that $\frac{\partial H}{\partial v}(\eta, v) \cdot(\eta, w)=$ $\left\langle e_{1}, w\right\rangle \forall w \in \mathbf{E}$. Similarly, setting $w=0$ yields

$$
\frac{\partial H}{\partial \eta}(\eta, v) \cdot(\eta, u)=-\left\langle e_{2}+\Gamma(\eta)\left(v, e_{1}\right), u\right\rangle \forall u \in \mathbf{E} .
$$

Thus, $H \in \mathcal{K}^{k}$.

Conversely, let $H \in \mathcal{K}^{k}$. Defining a vector field $\mathbf{X}_{H}$ by formula 4.3 and substituting into formula 4.4 one obtains for arbitrary vector $Z \in T_{(\eta, v)} T Q$

$$
\Omega\left(\mathbf{X}_{H}, Z\right)=\left\langle\frac{\partial H}{\partial v}, K Z\right\rangle+\left\langle\frac{\partial H}{\partial \eta}, T \tau Z\right\rangle=d H \cdot Z
$$

Proposition 4.6. Let $f, g \in \mathcal{K}^{k}$ be arbitrary. Then

$$
\{f, g\}=\Omega\left(\mathbf{X}_{f}, \mathbf{X}_{g}\right) .
$$

Proof. By Proposition 4.5, the vector fields $\mathbf{X}_{f}, \mathbf{X}_{g}$ are defined whenever $\{f, g\}$ is. Then

$$
\Omega\left(\mathbf{X}_{f}, \mathbf{X}_{g}\right)=d f \cdot \mathbf{X}_{g}=\frac{\partial f}{\partial \eta} \cdot T \tau \mathbf{X}_{g}+\frac{\partial f}{\partial v} K \mathbf{X}_{g}=\frac{\partial f}{\partial \eta} \cdot \frac{\partial g}{\partial v}-\frac{\partial g}{\partial \eta} \cdot \frac{\partial f}{\partial v}=\{f, g\}
$$

THEOREM 4.7. The bracket $\{\cdot, \cdot\}$ is antisymmetric, bilinear, derivation on each factor and makes $\mathcal{K}$ into a Lie-algebra.

Proof. Antisymmetry, linearity and property of being derivation follows directly from the definition of the bracket. By Theorem $4.3\{\cdot, \cdot\}$ leaves $\mathcal{K}$ invariant. Then, Jacobi identity follows from Proposition 4.6 in the usual way, for example as in Marsden and Ratiu [1999].

Now, $T Q$ has both symplectic and Poisson structures, and therefore two generally different definitions of Hamiltonian vector fields. We need to check that in our case these coincide. To do so, let $\mathbf{X}_{f}^{P}$ temporarily denote the Hamiltonian vector field with respect to 
Poisson structure $\{\cdot, \cdot\}$ and $\mathbf{X}_{f}$ denotes the Hamiltonian vector field with respect to canonical symplectic form corresponding to function the $f$. Recall, that $\mathbf{X}_{f}^{P}$ is defined as a vector field such that

Thus, for all $h \in \mathcal{K}$,

$$
\mathbf{X}_{f}^{P}[h]=\{h, f\} \quad \forall h \in \mathcal{K} .
$$

$$
\begin{aligned}
\mathbf{X}_{f}^{P}[h] & =\frac{\partial h}{\partial \eta} \cdot \frac{\partial f}{\partial v}-\frac{\partial h}{\partial v} \cdot \frac{\partial f}{\partial \eta} \\
& =d h \cdot \mathbf{X}_{f}^{P}=\frac{\partial h}{\partial \eta} \cdot T \tau \mathbf{X}_{f}^{P}+\frac{\partial h}{\partial v} \cdot K \mathbf{X}_{f}^{P}
\end{aligned}
$$

and therefore, $T \tau \mathbf{X}_{f}^{P}=\frac{\partial f}{\partial v}$ and $K \mathbf{X}_{f}^{P}=-\frac{\partial f}{\partial \eta}$. Comparing this with formula 4.3, we see that $\mathbf{X}_{f} \equiv \mathbf{X}_{f}^{P}$. Finally, from the coordinate expression, it is easy to see that $\mathbf{X}_{f}$ is a well defined $C^{k}$ vector field for any $f \in \mathcal{K}^{k}$.

Previously we established that classes $\mathcal{K}^{k}$ are preserved under bracketing. Unfortunately, for $f \in \mathcal{K}^{k}$ and a diffeomorphism $\psi: T Q \rightarrow T Q$ the composition $f \circ \psi$ does not have to be in any class $\mathcal{K}^{m}$. One can, however, compose with symplectic diffeomorphisms.

Proposition 4.8. Let $\psi$ be a symplectic $C^{k}$ diffeomorphism, $f \in \mathcal{K}^{k}$. Then $f \circ \psi \in \mathcal{K}^{k}$.

Proof. We have

$$
\mathbf{X}_{f \circ \psi}=\psi^{*}\left(\mathbf{X}_{f}\right)
$$

and so by Proposition 4.5, $f \circ \psi \in \mathcal{K}^{k}$.

Proposition 4.9. Let $F_{t}$ be a flow of a smooth Hamiltonian vector field on TQ. Then $F_{t}$ is a Poisson, i.e. for all $f, g \in \mathcal{K}$

$$
\left\{f \circ F_{t}, g \circ F_{t}\right\}=\{f, g\} \circ F_{t} .
$$

Proof. $F_{t}$ is symplectic with respect to the weak Riemannian form. Since $F_{t}$ preserves class $\mathcal{K}$, the statement follows from Jacobi identity by the usual argument.

\section{Geometric Properties of the Flow of the Euler Equations}

As we stated earlier, in Ebin and Marsden [1970] it is shown that $\mathcal{D}_{\mu}^{s}$ carries a smooth Riemannian connection, and therefore the results of the previous section apply. Therefore, by those results, the space $T \mathcal{D}_{\mu}^{s}$ carries a Poisson structure (in the precise sense given there) which we denote $\{\cdot, \cdot\}$. Let $K, \widehat{K}, \widetilde{K}$ stand for the corresponding connector maps on the underlying manifold $M$, on $\mathcal{D}^{s}$ and $\mathcal{D}_{\mu}^{s}$ respectively, while $\nabla, \hat{\nabla}, \widetilde{\nabla}$ are the corresponding connections and $\Gamma, \widehat{\Gamma}, \widetilde{\Gamma}$ are the corresponding Christoffel maps. In the following $\langle\cdot, \cdot\rangle$ denotes the Riemannian metric on $M, \mathcal{D}^{s}, \mathcal{D}_{\mu}^{s}$ and an induced scalar product on $\mathfrak{X}_{\text {div }}^{s}=T_{e} \mathcal{D}_{\mu}^{s}$ depending on the context. The relationship between these metrics is given by 2.2 .

Recall the notation from $\S 3$. Namely, let $F_{t}$ be the flow of the spray on $T \mathcal{D}_{\mu}^{s}, \tilde{F}_{t}$ denote the flow of Euler equation on $\mathfrak{X}_{\text {div }}^{s}$ and $\pi: T \mathcal{D}_{\mu}^{s} \rightarrow \mathfrak{X}_{\text {div }}^{s}, \pi(\eta, v)=v \circ \eta^{-1}$. Recall also that we have the commutative diagram

Proposition 5.1. The following diagram is commutative:

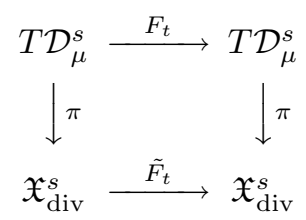

Now we prepare and recall from Ebin and Marsden [1970] some useful Lemmas. 
Lemma 5.2. Let $\xi \in \mathcal{D}_{\mu}^{s}$. Define $R_{\xi}: \mathcal{D}_{\mu}^{s} \rightarrow \mathcal{D}_{\mu}^{s}$ via $R_{\xi}(\eta)=\eta \circ \xi, \forall \xi$. Then

$$
T R_{\xi} \circ F_{t}(v)=F_{t} \circ T R_{\xi}(v) \quad \forall v \in T \mathcal{D}_{\mu}^{s} .
$$

Proof. Indeed, notice that

$$
\begin{aligned}
\frac{d}{d t}\left(\eta_{t} \circ \xi, \dot{\eta}_{t} \circ \xi\right) & =\left(\eta_{t} \circ \xi, \dot{\eta}_{t} \circ \xi, \dot{\eta}_{t} \circ \xi, \ddot{\eta}_{t} \circ \xi\right) \\
& =T T R_{\xi}\left(\eta_{t}, \dot{\eta}_{t}, \dot{\eta}_{t}, \ddot{\eta}_{t}\right)=T T R_{\xi} S\left(F_{t}(v)\right) \\
& =S\left(T R_{\xi} F_{t}(v)\right)=S\left(\eta_{t} \circ \xi, \dot{\eta}_{t} \circ \xi\right)
\end{aligned}
$$

by right invariance of the spray. Thus, $T R_{\xi} F_{t}(v)=\left(\eta_{t} \circ \xi, \dot{\eta}_{t} \circ \xi\right)$ is an integral curve of $S$. Since $T R_{\xi} F_{0}(v)=T R_{\xi}(v)$, the statement of the Lemma follows from uniqueness of integral curves.

Recall that by definition, $\tilde{F}_{t}(V)=\pi \circ F_{t}(V)$ for all $V \in T_{e} \mathcal{D}_{\mu}^{s}=\mathfrak{X}_{\text {div }}^{s}$. Let $V=(\eta, v) \in$ $T \mathcal{D}_{\mu}^{s}$. Then, using the preceding Lemma, we obtain

$$
\begin{aligned}
\tilde{F}_{t} \circ \pi(V) & =\pi \circ F_{t}(\pi(V)) \\
& =\pi \circ F_{t} \circ T R_{\eta^{-1}}(V)=\pi \circ T R_{\eta^{-1}} \circ F_{t}(V) .
\end{aligned}
$$

Notice, that $\pi \circ T R_{\xi}=\pi$ for any $\xi \in \mathcal{D}_{\mu}^{s}$. Indeed,

$$
\begin{aligned}
\pi \circ T R_{\xi}(\eta, v) & =\pi(\eta \circ \xi, v \circ \xi)=\left(e, v \circ \xi \circ(\eta \circ \xi)^{-1}\right) \\
& =\left(e, v \circ \xi \circ \xi^{-1} \circ \eta^{-1}\right)=\left(e, v \circ \eta^{-1}\right)=\pi(\eta, v) .
\end{aligned}
$$

Thus $\pi \circ T R_{\eta^{-1}}=\pi$ and the Proposition is proved.

A Poisson Structure on the Lie Algebra. Now, we construct a Poisson bracket $\{\cdot, \cdot\}_{+}$on $\mathfrak{X}_{\text {div }}^{s}$ so that $\pi$ is a Poisson map. For $f, g: \mathfrak{X}_{\text {div }}^{s} \rightarrow \mathbb{R}$ such that $d f, d g: \mathfrak{X}_{\text {div }}^{s} \rightarrow \mathfrak{X}_{\text {div }}^{r}$ define

$$
\{f, g\}_{+}(v)=\left\langle d g(v), \nabla_{d f(v)} v\right\rangle-\left\langle d f(v), \nabla_{d g(v)} v\right\rangle .
$$

As in $\S 4$, define

$$
\mathcal{K}^{k, s}=\left\{f \in C^{k+1}\left(\mathfrak{X}_{\mathrm{div}}^{s}, \mathbb{R}\right) \mid d f \in C^{k}\left(\mathfrak{X}_{\mathrm{div}}^{s}, \mathfrak{X}_{\mathrm{div}}^{s}\right)\right\}
$$

and

$$
\mathcal{K}_{r, t}^{k, s}=\left\{f \in C^{k}\left(\mathfrak{X}_{\text {div }}^{s}, \mathbb{R}\right) \mid d f \in C^{k}\left(\mathfrak{X}_{\text {div }}^{r}, \mathfrak{X}_{\text {div }}^{t}\right)\right\}
$$

ThEOREM 5.3. Let $s>n / 2+1$. Then $\{\cdot, \cdot\}_{+}$is a bilinear map $\mathcal{K}^{k, s} \times \mathcal{K}^{k, s} \rightarrow \mathcal{K}_{s+1, s-1}^{k, s}$ and a derivation on each factor. Moreover, it satisfies Jacobi identity on $\mathfrak{X}_{\mathrm{div}}^{s+1}$, that is for all $f, g, h \in \mathcal{K}^{k, s}$, and $v \in \mathfrak{X}_{\mathrm{div}}^{s+1}$,

$$
O(v):=\left\{f,\{g, h\}_{+}\right\}_{+}(v)+\left\{h,\{f, g\}_{+}\right\}_{+}(v)+\left\{g,\{h, f\}_{+}\right\}_{+}(v)=0
$$

Proof. Let $f, g \in \mathcal{K}^{k, s}$. Recall, that for $r>n / 2, H^{r}(M, \mathbb{R})$ is an algebra. Thus, $(u, v) \rightarrow \nabla_{u} v$ is a bilinear bounded map $\mathfrak{X}_{\text {div }}^{s} \times \mathfrak{X}_{\text {div }}^{s} \rightarrow \mathfrak{X}_{\text {div }}^{s-1}\left(\right.$ and $\mathfrak{X}_{\text {div }}^{s} \times \mathfrak{X}_{\text {div }}^{s+1} \rightarrow \mathfrak{X}_{\text {div }}^{s}$ ), hence smooth. This implies that

$$
z(v)=\{f, g\}_{+}(v) \in C^{k}\left(\mathfrak{X}_{\mathrm{div}}^{s}, \mathbb{R}\right)
$$

Bilinearity and derivation property of $\{\cdot, \cdot\}_{+}$trivially follows from properties of $d, \nabla$ and $\langle\cdot, \cdot\rangle$. 
Now we calculate $d z$. Let $v, u \in \mathfrak{X}_{\text {div }}^{s+1}$. Since $z \in C^{k}\left(\mathfrak{X}_{\text {div }}^{s}, \mathbb{R}\right)$, the Fréchet derivative of $z$ exists and coincides with its Gateaux derivative. Thus, by bilinearity of scalar product and $\nabla$

$$
\begin{aligned}
d z(v) \cdot u= & \left.\frac{d}{d t}\right|_{t=0} z(v+t u) \\
= & \left\langle D d g(v) \cdot u, \nabla_{d f(v)} v\right\rangle+\left\langle d g(v), \nabla_{D d f(v) \cdot u} v\right\rangle \\
& +\left\langle d g(v), \nabla_{d f(v)} u\right\rangle-\left\langle D d f(v) \cdot u, \nabla_{d g(v)} v\right\rangle \\
& -\left\langle d f(v), \nabla_{D d g(v) \cdot u} v\right\rangle-\left\langle d f(v), \nabla_{d g(v)} u\right\rangle
\end{aligned}
$$

Lemma 5.4. Let $X \in \mathfrak{X}_{\text {div }}^{s}, s>n / 2+1$, and let $Y, W$ be $H^{s}$ vector fields on $M$. Then

$$
\left\langle Y, \nabla_{X} W\right\rangle=-\left\langle\nabla_{X} Y, W\right\rangle .
$$

Proof. By the Sobolev theorems, $X$ is a $C^{1}$ vector field on $M$. By properties of the Riemannian connection, for all $m \in M$

$$
\left\langle Y, \nabla_{X} W\right\rangle_{m}=-\left\langle\nabla_{X} Y, W\right\rangle_{m}+X\langle Y, W\rangle_{m}
$$

Thus,

$$
\left\langle Y, \nabla_{X} W\right\rangle=-\left\langle\nabla_{X} Y, W\right\rangle+\int_{M} X\langle Y, W\rangle_{m} \mu .
$$

Let $G_{t}$ be a flow of $X$ on $M$. Since $X$ is divergence free, $\mu$ is $G_{t}$ invariant, i.e. $G_{t}^{*}(\mu)=\mu$, where $G_{t}^{*}$ denotes a pullback by $G_{t}$. Then

$$
\begin{aligned}
\int_{M} X\langle Y, W\rangle_{m} & =\int_{M} \frac{d}{d t}{ }_{t=0}\langle Y, W\rangle_{G_{t}(m)} \mu \\
& =\frac{d}{d t} \int_{t=0}\langle Y, W\rangle_{G_{t}(m)} G_{t}^{*}(\mu) \\
& =\frac{d}{d t} \int_{t=0} \int_{M} G_{t}^{*}\left(\langle Y, W\rangle_{m} \mu\right) \\
& =\frac{d}{d t} \int_{t=0}\langle Y, W\rangle_{m} \mu=0 .
\end{aligned}
$$

Lemma 5.5. Let $d f \in C^{k}\left(\mathfrak{X}_{\mathrm{div}}^{s}, \mathfrak{X}_{\mathrm{div}}^{t}\right), s, t \geq 0$. Then for all $u, v, w \in \mathfrak{X}_{\mathrm{div}}^{s}$

$$
\langle D d f(v) \cdot u, w\rangle=\langle D d f(v) \cdot w, u\rangle .
$$

Proof. We compute as follows:

$$
\begin{aligned}
\langle D d f(v) \cdot u, w\rangle & =\frac{d}{d t}_{t=0}\langle d f(v+t u), w\rangle \\
& =\frac{d}{d t}_{t=0} \frac{d}{d s}_{s=0} f(v+t u+s w) \\
& =\frac{d}{d s}{ }_{s=0} \frac{d}{d t}_{t=0} f(v+t u+s w) \\
& =\langle D d f(v) \cdot w, u\rangle .
\end{aligned}
$$

Lemma 5.6. (The Hodge Decomposition; see Ebin and Marsden [1970]). Let X be an $H^{s}$ vector field on $M, s \geq 0$. There is an $H^{s+1}$ function $\theta$ and an $H^{s}$ vector field $Y$ with $Y$ divergence free, such that

$$
X=\operatorname{grad} \theta+Y
$$

Further, the projection maps

$$
\begin{gathered}
P_{e}(X)=Y \\
Q(X)=\operatorname{grad} \theta
\end{gathered}
$$


are continuous linear maps on $H^{s}(M, T M)$. The decomposition is orthogonal in $L^{2}$ sense, that is for all $Z \in \mathfrak{X}_{\mathrm{div}}^{s}$

$$
\langle Z, X\rangle=\langle Z, Y\rangle=\left\langle Z, P_{e} X\right\rangle
$$

LEMmA 5.7. There is a bilinear continuous map $B: \mathfrak{X}_{\mathrm{div}}^{s} \times \mathfrak{X}_{\mathrm{div}}^{s+1} \rightarrow \mathfrak{X}_{\mathrm{div}}^{s}(s>n / 2)$ such that for all $Z \in \mathfrak{X}_{\mathrm{div}}^{s}, W \in X^{s+1}, Y \in C(M, T M)$

$$
\left\langle Z, \nabla_{Y} W\right\rangle=\langle B(Z, W), Y\rangle
$$

Proof. Fix coordinate system $\left\{x_{i}\right\}$ on $M$ and let $g_{i j}$ denote components of metric tensor, $Z^{i}$ denote components of vector field $Z$ in the chosen system. Let $g_{i j} g^{j k}=\delta_{i}^{k}$ (as usually, the summation on repeated indexes is understood). Then

$$
\begin{aligned}
\left\langle Z, \nabla_{Y} W\right\rangle & =\int_{M} g_{i j} Z^{i}\left(\frac{\partial W^{j}}{\partial x_{k}} Y^{k}+\Gamma_{k r}^{j} Y^{k} W^{r}\right) \mu \\
& =\int_{M} g_{s m} g^{m k} g_{i j} Z^{i}\left(\frac{\partial W^{j}}{\partial x_{k}}+\Gamma_{k r}^{j} W^{r}\right) Y^{s} \mu \\
& =\langle V, Y\rangle
\end{aligned}
$$

where

$$
V^{m}=g^{m k} g_{i j} Z^{i}\left(\frac{\partial W^{j}}{\partial x_{k}}+\Gamma_{k r}^{j} W^{r}\right) .
$$

Since $H^{s}$ is an algebra for $s>n / 2$ it follows that $V$ is an $H^{s}$ vector field. Now we set

$$
B(Z, W)=P_{e} V
$$

and use 5.1.

By Lemmata 5.4-5.7, we have

$$
\begin{aligned}
d z(v) \cdot u= & \left\langle D d g(v) \cdot P_{e} \nabla_{d f(v)} v, u\right\rangle+\langle D d f(v) \cdot B(d g(v), v), u\rangle+\left\langle\nabla_{d f(v)} d g(v), u\right\rangle \\
& -\left\langle D d f(v) \cdot P_{e} \nabla_{d g(v)} v, u\right\rangle+\langle D d g(v) \cdot B(d f(v), v), u\rangle+\left\langle\nabla_{d g(v)} d f(v), u\right\rangle .
\end{aligned}
$$

Thus for any $v \in \mathfrak{X}_{\text {div }}^{s+1}$,

$$
\begin{aligned}
d\{f, g\}_{+}(v)= & P_{e}\left[\nabla_{d g(v)} d f(v)-\nabla_{d f(v)} d g(v)\right] \\
& +D d f(v) \cdot B(d g(v), v)-D d g(v) \cdot B(d f(v), v) \\
& +D d g(v) \cdot P_{e} \nabla_{d f(v)} v-D d f(v) \cdot P_{e} \nabla_{d g(v)} v
\end{aligned}
$$

and hence $d\{f, g\}_{+} \in C^{k}\left(\mathfrak{X}_{\mathrm{div}}^{s+1}, \mathfrak{X}_{\mathrm{div}}^{s-1}\right)$ and $\{f, g\}_{+} \in \mathcal{K}_{s+1, s-1}^{k, s}$.

Remark. If $f, g \in \mathcal{K}^{k, s} \cap \mathcal{K}^{k, s+1}$, then $v \rightarrow P_{e} \nabla_{d f(v)} d g(v), v \rightarrow P_{e} \nabla_{d g(v)} d f(v)$ are $C^{k}$ as maps $\mathfrak{X}_{\text {div }}^{s+1} \rightarrow \mathfrak{X}_{\text {div }}^{s}$, hence $\{f, g\}_{+} \in \mathcal{K}_{s+1, s}^{k, s}$.

Now we prove the Jacobi identity. To simplify notation, we set

$$
B_{f}(v)=B(d f(v), v), \quad \nabla_{f}(v)=P_{e} \nabla_{d f(v)} v
$$


Moreover, since in the following argument all functions are evaluated at the same point $v \in \mathfrak{X}_{\text {div }}^{s+1}$, we will write $B_{f}, \nabla_{f}, d f$ instead of $B_{f}(v)$, etc. By Lemmata 5.5-5.7, we obtain

$$
\begin{aligned}
O_{f g h}(v)= & \left\{f,\{g, h\}_{+}\right\}_{+}(v) \\
= & \left\langle d\{g, h\}_{+}, \nabla_{f}\right\rangle-\left\langle B_{f}, d\{g, h\}_{+}\right\rangle \\
= & \left\langle d\{g, h\}_{+}, \nabla_{f}-B_{f}\right\rangle \\
= & \left\langle P_{e}\left[\nabla_{d h} d g-\nabla_{d g} d h\right], \nabla_{f}-B_{f}\right\rangle+\left\langle D d g \cdot\left(B_{h}-\nabla_{h}\right), \nabla_{f}-B_{f}\right\rangle \\
& \quad+\left\langle D d h \cdot\left(\nabla_{g}-B_{g}\right), \nabla_{f}-B_{f}\right\rangle \\
= & \left\langle[d h, d g], \nabla_{f}-B_{f}\right\rangle+D_{g h f}-D_{h f g},
\end{aligned}
$$

where $D_{g h f}=\left\langle D d g \cdot\left(B_{h}-\nabla_{h}\right), \nabla_{f}-B_{f}\right\rangle$ and $[\cdot, \cdot]$ is a Lie bracket of vector fields on $M$. Notice that Lie bracket of divergence free vector fields is divergence free.

For $s>n / 2+2$

$$
\left\langle[d h, d g], \nabla_{f}-B_{f}\right\rangle=\langle[[d h(v), d g(v)], d f(v)], v\rangle .
$$

Since terms of type $D_{f g h}$ cancel out in the Jacobi cycle

$$
O(v)=O_{f g h}(v)+O_{h f g}(v)+O_{g h f}(v),
$$

and so the Jacobi identity for bracket $\{\cdot, \cdot\}_{+}$follows from the Jacobi identity for vector fields. However, for $n / 2+1<s \leq n / 2+2$ Lie bracket of $d h(v)$ and $d g(v)$ is an $\mathfrak{X}_{\text {div }}^{s-1}$ vector field, hence merely continuous and therefore $[[d h(v), d g(v)], d f(v)]$ may fail to exist. Therefore, in this case more care is needed.

Let

$$
\begin{gathered}
A_{f g h}=\left\langle d f, \nabla_{d g} \nabla_{d h} v\right\rangle, \\
C_{f g h}=\left\langle d f, \nabla_{[d g, d f]} v\right\rangle
\end{gathered}
$$

With this notation in mind, by Lemma 5.4 and the Hodge decomposition

$$
\left\langle[d h, d g], \nabla_{f}\right\rangle=\left\langle\nabla_{d h} d g-\nabla_{d g} d h, \nabla_{d f} v\right\rangle=-A_{g h f}+A_{h g f} .
$$

Similarly, by definition of $B$

$$
\left\langle[d h, d g], B_{f}\right\rangle=C_{f h g} .
$$

By a well known formula for Riemannian connection,

$$
\nabla_{X} \nabla_{Y} Z-\nabla_{Y} \nabla_{X} Z=\nabla_{[X, Y]} Z,
$$

for all sufficiently smooth vector fields $X, Y, Z$. Thus,

$$
A_{f g h}-A_{f h g}=\left\langle d f, \nabla_{d g} \nabla_{d h} v-\nabla_{d h} \nabla_{d f} v\right\rangle=\left\langle d f, \nabla_{[d g, d h]} v\right\rangle=C_{f g h} .
$$

Thus,

$$
\left\{f,\{g, h\}_{+}\right\}_{+}=-A_{g h f}+A_{h g f}-C_{f h g}+D_{g h f}-D_{h f g},
$$

and so

$$
\begin{gathered}
\left\{f,\{g, h\}_{+}\right\}_{+}+\left\{h,\{f, g\}_{+}\right\}_{+}+\left\{g,\{h, f\}_{+}\right\}_{+} \\
=-A_{g h f}+A_{h g f}-C_{f h g}+D_{g h f}-D_{h f g} \\
\quad-A_{f g h}+A_{g f h}-C_{h g f}+D_{f g h}-D_{g h f} \\
\quad-A_{h f g}+A_{f h g}-C_{g f h}+D_{h f g}-D_{f g h} \\
=\left(A_{g f h}-A_{g h f}-C_{g f h}\right)+\left(A_{f h g}-A_{f g h}-C_{f h g}\right) \\
+\left(A_{h g f}-A_{h f g}-C_{h g f}\right)=0 .
\end{gathered}
$$


Remark. If $d f(v), d h(v) \in \mathfrak{X}_{\text {div }}^{s}, s>n / 2+1$, then by Lemma 5.4

$$
\{f, h\}_{+}(v)=\langle[d h(v), d f(v)], v\rangle .
$$

This shows that bracket $\{\cdot, \cdot\}_{+}$is naturally related to Lie-Poisson bracket on $\left(\mathfrak{X}_{\text {div }}^{s}\right)^{*}$.

Now we establish the relationship between Poisson bracket $\{\cdot, \cdot\}_{+}$on $\mathfrak{X}_{\text {div }}^{s}$ that we just introduced and Poisson bracket $\{\cdot, \cdot\}$ on $\mathcal{D}_{\mu}^{s}$. For $f, h: \mathfrak{X}_{\text {div }}^{s} \rightarrow \mathbb{R}$ define

$$
f_{R}=f \circ \pi \text {. }
$$

THEOREM 5.8. Define the function spaces

$$
C_{r}^{k}\left(\mathfrak{X}_{\mathrm{div}}^{s}\right)=\left\{f \in C^{k}\left(\mathfrak{X}_{\mathrm{div}}^{s}, \mathbb{R}\right) \mid d f(v) \in \mathfrak{X}_{\mathrm{div}}^{r} \forall v \in \mathfrak{X}_{\mathrm{div}}^{s}\right\},
$$

and

$$
C_{r}^{k}\left(T \mathcal{D}_{\mu}^{s}\right)=\left\{f \in C^{k}\left(T \mathcal{D}_{\mu}^{s}, \mathbb{R}\right) \mid \frac{\partial f}{\partial \eta}(v), \frac{\partial f}{\partial v}(v) \in T \mathcal{D}_{\mu}^{r} \forall v \in T \mathcal{D}_{\mu}^{s}\right\} .
$$

Then $f_{R} \in C_{r}^{k}\left(T \mathcal{D}_{\mu}^{s+k}\right)$ for $f \in C_{r}^{k}\left(\mathfrak{X}_{\mathrm{div}}^{s}\right)(r, s>n / 2+1, k \geq 1)$ and for all $f, h \in$ $C_{r}^{1}\left(X^{s}\right), v \in \mathfrak{X}_{\operatorname{div}}^{s+1}$

$$
\{f, h\}_{+}(v)=\left\{f_{R}, h_{R}\right\}(v)=\{f \circ \pi, g \circ \pi\}(v) .
$$

Proof. Without loss of generality $s \geq r$. Since $\pi$ is not even a $C^{1}$ function $\mathcal{D}_{\mu}^{s} \rightarrow \mathfrak{X}_{\text {div }}^{s}$ it is not obvious that $\left\{f_{R}, h_{R}\right\}$ is defined. However, differentiating $f_{R}$ and $h_{R}$ as functions $T \mathcal{D}_{\mu}^{s+k} \rightarrow T \mathcal{D}_{\mu}^{s}$ one obtains the required result.

LEMma 5.9. Under the assumptions of the Theorem,

$$
\frac{\partial f_{R}}{\partial v}(\eta, v)=T R_{\eta} d f(\pi(\eta, v))
$$

Proof. It is well known (Ebin and Marsden [1970]) that $\pi \in C^{k}\left(T \mathcal{D}_{\mu}^{s+k}, T \mathcal{D}_{\mu}^{s}\right)$. Notice, that for $(\eta, u) \in T \mathcal{D}_{\mu}^{s+k}$,

$$
\frac{\partial \pi}{\partial v}(\eta, v) \cdot(\eta, u)=\frac{d}{d t}_{t=0} \pi(\eta, v+t u)=\left(e, v \circ \eta^{-1}, 0, u \circ \eta^{-1}\right)
$$

where time derivative is taken in $T \mathcal{D}_{\mu}^{s}$. By lemma 4.1

$$
\frac{\partial f_{R}}{\partial v}=d f \cdot \widetilde{K} \frac{\partial \pi}{\partial v} .
$$

Thus, by right invariance of the metric on $\mathcal{D}_{\mu}^{s}$

$$
\begin{aligned}
\frac{\partial f_{R}}{\partial v}(\eta, v) \cdot(\eta, u) & =d f\left(v \circ \eta^{-1}\right) \cdot \widetilde{K}\left(e, v \circ \eta^{-1}, 0, u \circ \eta^{-1}\right) \\
& =d f\left(v \circ \eta^{-1}\right) \cdot\left(u \circ \eta^{-1}\right) \\
& =\left\langle d f\left(\pi(\eta, v), u \circ \eta^{-1}\right\rangle_{e}\right. \\
& =\left\langle T R_{\eta} d f(\pi(\eta, v)),(\eta, u)\right\rangle_{\eta} .
\end{aligned}
$$

LEMMA 5.10. Under the assumptions of the Theorem

$$
\frac{\partial f_{R}}{\partial \eta}(\eta, v) \cdot(\eta, u)=-\left\langle d f\left(v \circ \eta^{-1}\right), \widetilde{K}\left[T\left(v \circ \eta^{-1}\right) \circ\left(u \circ \eta^{-1}\right)\right]\right\rangle_{e}
$$

that is,

$$
\frac{\partial f_{R}}{\partial \eta}(\eta, v)=-T R_{\eta} B_{f}(\pi(\eta, v))
$$


Proof. First, we calculate $\frac{\partial \pi}{\partial \eta}$. Let $(\eta, u) \in T \mathcal{D}_{\mu}^{s+k},\left(\eta_{t}, v_{t}\right)$ be a parallel translation of $(\eta, v)$ with $\frac{d}{d t}{ }_{t=0} \eta_{t}=u$. Recall that

$$
\frac{d}{d t} \eta_{t}^{-1}=-T \eta_{t}^{-1} \circ \frac{d}{d t} \eta_{t} \circ \eta_{t}^{-1}
$$

Then, by Lemma 4.2,

$$
\begin{aligned}
\frac{\partial \pi}{\partial \eta}(\eta, v) \cdot(\eta, u) & =\frac{d}{d t}_{t=0} \pi\left(\eta_{t}, v_{t}\right)=\frac{d}{d t} v_{t=0} \circ \eta_{t}^{-1} \\
& =T v_{0} \circ \frac{d}{d t} \eta_{t=0}^{-1}+\left(\frac{d}{d t}_{t=0} v_{t}\right) \circ \eta_{0}^{-1} \\
& =-T v \circ T \eta^{-1} \circ u \circ \eta^{-1}-\left(\frac{d}{d t}_{t=0} v_{t}\right) \circ \eta^{-1}
\end{aligned}
$$

Since connection on $\mathcal{D}_{\mu}^{s}$ is right invariant, i.e.,

$$
\widetilde{K} \circ T T R_{\xi}=T R_{\xi} \circ \widetilde{K} \quad \forall \xi \in \mathcal{D}_{\mu}^{s}
$$

we have

$$
\widetilde{K}\left[\frac{d}{d t}_{t=0} v_{t} \circ \eta^{-1}\right]=\left[\widetilde{K} \frac{d}{d t}{ }_{t=0} v_{t}\right] \circ \eta^{-1}=0
$$

By Lemma 4.1

$$
\frac{\partial f_{R}}{\partial \eta}=d f \cdot \widetilde{K} \frac{\partial \pi}{\partial \eta}
$$

Combining above equalities together, we get

$$
\begin{gathered}
\frac{\partial f_{R}}{\partial \eta}(\eta, v) \cdot(\eta, u)=-d f \cdot \widetilde{K}\left[T\left(v \circ \eta^{-1}\right) \circ\left(u \circ \eta^{-1}\right)\right] \\
=-\left\langle d f\left(v \circ \eta^{-1}\right), \widetilde{K}\left[T\left(v \circ \eta^{-1}\right) \circ\left(u \circ \eta^{-1}\right)\right]\right\rangle_{e} .
\end{gathered}
$$

We claim that for all $X, Y, Z \in \mathfrak{X}_{\text {div }}^{s}$

$$
\langle Z, \widetilde{K}[T X \circ Y]\rangle=\left\langle Z, \nabla_{Y} X\right\rangle .
$$

Recall that by construction (see Ebin and Marsden [1970]),

$$
\begin{gathered}
\widetilde{K}=P \circ \widehat{K}, \\
P=T R_{\eta} \circ P_{e} \circ T R_{\eta}^{-1}, \\
\widehat{K}(Y)=K \circ Y,
\end{gathered}
$$

By a well known formula of differential geometry, we have

$$
K \circ T X \circ Y=\nabla_{Y} X
$$

and hence

$$
\widetilde{K}[T X \circ Y]=P_{e}\left[\nabla_{Y} X\right]
$$

By the Hodge decomposition

$$
\langle Z, \widetilde{K}[T X \circ Y]\rangle=\left\langle Z, \nabla_{Y} X\right\rangle=\langle B(Z, X), Y\rangle .
$$

By the above developments and right invariance of metric on $\mathcal{D}_{\mu}^{s}$, we have

$$
\frac{\partial f_{R}}{\partial \eta}(\eta, v) \cdot(\eta, u)=-\left\langle B_{f}\left(v \circ \eta^{-1}\right), u \circ \eta^{-1}\right\rangle=-\left\langle T R_{\eta} B_{f}(\pi(\eta, v)), u\right\rangle_{\eta} .
$$


Calculating $\left\{f_{R}, h_{R}\right\}$ at $v \in \mathfrak{X}_{\text {div }}^{s+1}$ by Lemmata 5.9,5.10, we obtain

$$
\begin{aligned}
\left\{f_{R}, h_{R}\right\}(v) & =-\left\langle B_{f}(v), d h(v)\right\rangle+\left\langle B_{h}(v), d f(v)\right\rangle \\
& =-\left\langle d f(v), \nabla_{d h(v)} v\right\rangle+\left\langle d h(v), \nabla_{d f(v)} v\right\rangle \\
& =\{d f, d h\}_{+}(v) .
\end{aligned}
$$

Proposition 5.11. Map $\pi: T \mathcal{D}_{\mu}^{s} \rightarrow \mathfrak{X}_{\text {div }}^{s}$ is a Poisson map, i.e. for all $f, h \in C_{r}^{1}\left(\mathfrak{X}_{\text {div }}^{s}\right)$ pointwise in $\operatorname{TD}_{\mu}^{s+1}(r, s>n / 2+1)$

$$
\{f \circ \pi, h \circ \pi\}=\{f, h\}_{+} \circ \pi .
$$
5.8 .

Proof. Since $\pi$ is the identity on $\mathfrak{X}_{\text {div }}^{s}$, the statement follows immediately from Theorem

Proposition 5.12. Let $v \in T \mathcal{D}_{\mu}^{r}$ and $f, g \in C^{1}\left(T \mathcal{D}_{\mu}^{r}, \mathbb{R}\right)$ are such that $\frac{\partial f}{\partial v}\left(F_{t}(v)\right)$, $\frac{\partial f}{\partial \eta}\left(F_{t}(v)\right), \frac{\partial g}{\partial v}\left(F_{t}(v)\right), \frac{\partial g}{\partial \eta}\left(F_{t}(v)\right) \in T \mathcal{D}_{\mu}^{s}, r, s>n / 2+1$. Then

$$
\left\{f \circ F_{t}, g \circ F_{t}\right\}(v)=\{f, g\}\left(F_{t}(v)\right) .
$$

In particular, $F_{t}$ preserves $C_{s}^{1}\left(T \mathcal{D}_{\mu}^{r}\right)$ and for $f, h \in \mathcal{K}^{1, s}$ pointwise in $T \mathcal{D}_{\mu}^{s+1}$

$$
\left\{f \circ \pi \circ F_{t}, h \circ \pi \circ F_{t}\right\}(v)=\{f \circ \pi, h \circ \pi\}\left(F_{t}(v)\right) .
$$

Proof. Without loss of generality $r \geq s$. First, we notice that covariant partial derivatives of $f \circ F_{t}, g \circ F_{t}$ at $v$ are elements of $T \mathcal{D}_{\mu}^{s}$. Indeed,

$$
\frac{\partial}{\partial \eta}\left(g \circ F_{t}\right)(v) \cdot u=\left\langle\frac{\partial g}{\partial \eta}\left(F_{t}(v)\right), T \widetilde{\tau} \frac{\partial F_{t}}{\partial \eta}(v) \cdot u\right\rangle+\left\langle\frac{\partial g}{\partial v}\left(F_{t}(v)\right), \widetilde{K} \frac{\partial F_{t}}{\partial \eta}(v) \cdot u\right\rangle .
$$

There is a function $\tilde{g} \in \mathcal{K}\left(T \mathcal{D}_{\mu}^{s}\right)$ such that

$$
\frac{\partial g}{\partial v}\left(F_{t}(v)\right)=\frac{\partial \tilde{g}}{\partial v}\left(F_{t}(v)\right), \quad \frac{\partial g}{\partial \eta}\left(F_{t}(v)\right)=\frac{\partial \tilde{g}}{\partial \eta}\left(F_{t}(v)\right)
$$

Thus,

$$
\frac{\partial}{\partial \eta}\left(g \circ F_{t}\right)(v) \cdot u=\frac{\partial}{\partial \eta}\left(\tilde{g} \circ F_{t}\right)(v) \cdot u
$$

However, by Proposition $4.8 \tilde{g} \circ F_{t} \in \mathcal{K}\left(T \mathcal{D}_{\mu}^{s}\right)$ for any $\tilde{g} \in \mathcal{K}\left(T \mathcal{D}_{\mu}^{s}\right)$, hence there is $Z_{g} \in$ $C^{\infty}\left(T \mathcal{D}_{\mu}^{s}, T \mathcal{D}_{\mu}^{s}\right)$ such that for all $u$,

$$
\frac{\partial}{\partial \eta}\left(g \circ F_{t}\right)(v) \cdot u=\frac{\partial}{\partial \eta}\left(\tilde{g} \circ F_{t}\right)(v) \cdot u=\left\langle Z_{g}(v), u\right\rangle .
$$

In a similar sense, one shows that $\frac{\partial}{\partial v}\left(f \circ F_{t}\right)(v) \in T \mathcal{D}_{\mu}^{s}$.

Thus, $\left\{f \circ F_{t}, g \circ F_{t}\right\}(v)$ is well defined and depends only on values of $\frac{\partial f}{\partial v}, \frac{\partial f}{\partial \eta}, \frac{\partial g}{\partial \eta}, \frac{\partial g}{\partial v}$ calculated at point $F_{t}(v)$. However, $\{f, g\} \circ F_{t}(v)$ also depends only on values of covariant partial derivatives at $F_{t}(v)$. Then, we choose $\tilde{f}, \tilde{g} \in \mathcal{K}\left(T \mathcal{D}_{\mu}^{s}\right)$ such that

$$
\begin{aligned}
& \frac{\partial f}{\partial v}\left(F_{t}(v)\right)=\frac{\partial \tilde{f}}{\partial v}\left(F_{t}(v)\right) \\
& \frac{\partial f}{\partial \eta}\left(F_{t}(v)\right)=\frac{\partial \tilde{f}}{\partial \eta}\left(F_{t}(v)\right), \\
& \frac{\partial g}{\partial v}\left(F_{t}(v)\right)=\frac{\partial \tilde{g}}{\partial v}\left(F_{t}(v)\right), \\
& \frac{\partial g}{\partial \eta}\left(F_{t}(v)\right)=\frac{\partial \tilde{g}}{\partial \eta}\left(F_{t}(v)\right) .
\end{aligned}
$$


The equality

$$
\left\{\tilde{f} \circ F_{t}, \tilde{g} \circ F_{t}\right\}(v)=\{\tilde{f}, \tilde{g}\} \circ F_{t}(v)
$$

follows from Proposition 4.9. By the preceding arguments, the same holds if we replace $\tilde{f}, \tilde{g}$ with $f, g$. This concludes the first part of the Proposition. The second part then follows.

Theorem 5.13. The map $\tilde{F}_{t}$ is Poisson with respect to the bracket $\{\cdot, \cdot\}_{+}$.

Proof. Let $f, h \in \mathcal{K}^{k, s}$. Then $f \circ \pi \in C_{s}^{1}\left(\mathfrak{X}_{\text {div }}^{s+1}\right)$. By Proposition 5.12.

$$
f \circ \tilde{F}_{t}=f \circ \pi \circ F_{t} \in C_{s}^{1}\left(\mathfrak{X}_{\mathrm{div}}^{s+1}\right)
$$

and we have pointwise in $\mathfrak{X}_{\text {div }}^{s+2}$ :

$$
\begin{aligned}
&\left\{f \circ \tilde{F}_{t}, h \circ \tilde{F}_{t}\right\}_{+} \quad(\text { Theorem 5.8) } \\
&=\left\{f \circ \tilde{F}_{t} \circ \pi, h \circ \tilde{F}_{t} \circ \pi\right\} \quad \text { (Proposition 5.1) } \\
&=\left\{f \circ \pi \circ F_{t}, h \circ \pi \circ F_{t}\right\} \quad \text { (Proposition 5.12) } \\
&=\{f \circ \pi, h \circ \pi\} \circ F_{t} \quad(\text { Proposition 5.11) } \\
&=\{f, h\}_{+} \circ \pi \circ F_{t}=\{f, h\}_{+} \circ \tilde{F}_{t} .
\end{aligned}
$$

\section{Conclusions}

In the previous sections we successfully implemented a nonsmooth Lie-Poisson reduction technique for the study of the Euler equations of ideal fluid flow. This enabled us to find a precise sense in which the flow of Euler equation on the Lie algebra of divergence free vector fields (parallel to the boundary of the fluid region) is a Hamiltonian system in the Poisson sense and that the flow consists of Poisson maps, despite the fact that this flow is believed (as maps from $H^{s}$ to $H^{s}$ ) to be continuous, but not differentiable.

A key part of this process was to introduce a Poisson structure on the space of divergence free vector fields. As one would expect from the bracket derived via a type of Lie-Poisson reduction, this bracket is closely related to the formal Lie-Poisson bracket on the dual to the Lie algebra of divergence free vector fields.

Even though we consider only Euler's equation, the technique developed here is directly applicable to several other important systems - those which can be written as an ODE on groups of diffeomorphisms, such as the following:

(1) The Camassa-Holm $(\mathrm{CH})$ equation on $S^{1}$ — see Camassa and Holm [1993]:

$$
u_{t}-u_{t x x}=-3 u u_{x}+2 u_{x} u_{x x}+u u_{x x x} .
$$

(2) The averaged Euler equations (or the LAE- $\alpha$ equations) — see Holm, Marsden, and Ratiu [1998a,b]:

$$
\partial_{t}\left(1-\alpha^{2} \Delta\right) u+(u \cdot \nabla)\left(1-\alpha^{2} \Delta\right) u-\alpha^{2}(\nabla u)^{T} \cdot \Delta u=-\operatorname{grad} p,
$$

where $\operatorname{div} u=0$ and $u$ satisfies appropriate boundary conditions, such as the no-slip conditions $u=0$ on $\partial M$.

(3) The EPDiff equation (also called the averaged template matching equation) on a compact manifold $M$ - see Holm and Marsden [2003] and Hirani, Marsden, and Arvo [2001]:

$$
\begin{aligned}
u_{t}-\alpha^{2} \Delta & u+u(\operatorname{div} u)-\alpha^{2}(\operatorname{div} u) \Delta u+(u \cdot \nabla) u \\
& -\alpha^{2}(u \cdot \nabla) \Delta u+(D u)^{T} \cdot u-\alpha^{2}(D u)^{T} \cdot \Delta u=0
\end{aligned}
$$

with appropriate boundary conditions, such as the no-slip conditions $u=0$ on $\partial M$. The EPDiff equations reduce to the $\mathrm{CH}$ equations in the case $M=S^{1}$. 
These equations may be derived as the right reduction to the identity of the geodesic motion on the appropriate Lie group (see, for example, Camassa and Holm [1993] and Misiolek [1998, 2002] for the case of the $\mathrm{CH}$ equations), and the preceding references for the other equations. The crucial technical fact that enables our methods to work in both cases is the smoothness of the spray on the Lie group. For the case of the CH equations and the LAE- $\alpha$ equations on regions with no boundary, this is due to Shkoller [1998] and for regions with boundary to Marsden, Ratiu, and Shkoller [2000]. For the case of the EPDiff equations, a rather convincing plausibility argument is given Holm and Marsden [2003].

One important direction in which we would like to pursue these ideas is that of nonsmooth solutions. Even for the ideal Euler equations, this is interesting because of the singular solutions, such as point vortices, vortex filaments and sheets. They clearly have themselves an interesting Poisson structure, as was investigated by Marsden and Weinstein [1983] and Langer and Perline [1991]. There are similar interesting singular solutions for the EPDiff equations, whose geometry is investigated in Holm and Marsden [2003]. It would be very interesting if, on the smaller spaces appropriate for these classes of singular solutions that are introduced in these references, the smooth spray property still holds and, if that is the case, whether or not one could then carry out the program in the present paper.

Another interesting direction for the present research is to the case of free boundary problems, a notoriously difficult case for infinite dimensional Poisson structures, even at the formal level (see Lewis, Marsden, Montgomery, and Ratiu [1986], Kruse, Marsden, and Scheurle [1993], Kruse, Mahalov, and Marsden [1999] and Bering [2000].)

\section{References}

Arnold, V. I. [1966], Sur la géométrie differentielle des groupes de Lie de dimenson infinie et ses applications à l'hydrodynamique des fluids parfaits, Ann. Inst. Fourier, Grenoble 16, 319-361.

Arnold, V. I. and B. Khesin [1998], Topological Methods in Hydrodynamics, volume 125 of Appl. Math. Sciences. Springer-Verlag.

Bering, K. [2000], Putting an edge on the Poisson bracket, J. Math. Phys. 41, 7468-7500.

Camassa, R. and D. D. Holm [1993], An integrable shallow water equation with peaked solitons, Phys. Rev. Lett. 71, 1661-1664.

Chernoff, P. R. and J. E. Marsden [1974], Properties of Infinite Dimensional Hamiltonian systems, volume 425 of Lecture Notes in Math. Springer, New York.

Do Carmo, M. [1992], Rimannian geometry, Birkhauser, Boston.

Ebin, D. G. and J. E. Marsden [1970], Groups of diffeomorphisms and the motion of an incompressible fluid, Ann. of Math. 92, 102-163.

Eliasson, H. [1967], Geometry of manifolds of maps, J. Differential Geometry 1, 169-194.

Hirani, A., J. E. Marsden, and J. Arvo [2001], Averaged template matching equations, Springer Lecture Notes in Computer Science 2134, 528-543.

Holm, D.D. and J. E. Marsden [2003], Momentum maps and measure valued solutions (peakons, filaments, and sheets) of the Euler-Poincaré equations for the diffeomorphism group. In Marsden, J. E. and T. S. Ratiu, editors, Festshrift for Alan Weinstein (to appear). Birkhäuser Boston.

Holm, D. D., J. E. Marsden, and T. S. Ratiu [1998a], Euler-Poincaré models of ideal fluids with nonlinear dispersion, Phys. Rev. Lett. 349, 4173-4177.

Holm, D. D., J. E. Marsden, and T. S. Ratiu [1998b], The Euler-Poincaré equations and semidirect products with applications to continuum theories, Adv. in Math. 137, 1-81.

Kato, T. [2000], On the smoothness of trajectories in incompressible perfect fluids, Cont. Math., Am. Math. Soc. 263, 109-130.

Kruse, H. P., A. Mahalov, and J. E. Marsden [1999], On the Hamiltonian structure and three-dimensional instabilities of rotating liquid bridges, Fluid Dyn. Research 24, 37-59. 
Kruse, H. P., J. E. Marsden, and J. Scheurle [1993], On uniformly rotating fluid drops trapped between two parallel plates, Lect. in Appl. Math., AMS 29, 307-317.

Langer, J. and R. Perline [1991], Poisson geometry of the filament equation, J. of Nonlinear Sci. 1, 71-94.

Lewis, D., J. E. Marsden, R. Montgomery, and T. S. Ratiu [1986], The Hamiltonian structure for dynamic free boundary problems, Physica D 18, 391-404.

Marsden, J. E. and T. S. Ratiu [1999], Introduction to Mechanics and Symmetry, volume 17 of Texts in Applied Mathematics, vol. 17; 1994, Second Edition, 1999. Springer-Verlag.

Marsden, J. E., T. Ratiu, and S. Shkoller [2000], The geometry and analysis of the averaged Euler equations and a new diffeomorphism group, Geom. Funct. Anal. 10, 582-599.

Marsden, J. E. and A. Weinstein [1983], Coadjoint orbits, vortices and Clebsch variables for incompressible fluids, Physica D 7, 305-323.

Misiolek, G. [1998], A shallow water equation as a geodesic flow on the Bott-Virasoro group, J. Geom. Phys. 24, 203-208.

Misiolek, G. [2002], Classical solutions of the periodic camassa-holm equation, Geom. Func. Anal. 12, 1080-1104.

Shkoller, S. [1998], Geometry and curvature of diffeomorphism groups with $H^{1}$ metric and mean hydrodynamics, J. Funct. An. 160, 337-365.

Départment de Mathématiques, École Polythechnique Fédérale de Lausanne, CH-1015 LauSANNE, SWITZERLAND

E-mail address: sergiy.vasylkevych@epfl.ch

Control and Dynamical Systems Department, 107-81, California Institute of Technology, PASAdena, CA 91125

E-mail address: marsden@cds.caltech.edu 